\title{
REFLEXIONES SOBRE LA PRÁCTICA CONTABLE EN EL PERÚ
}

\author{
REFLECTIONS ON THE COUNTABLE PRACTICE IN PERU
}

\author{
César Urbano Ventocilla* \\ Docente Asociado de la Facultad de Ciencias Contables, UNMSM
}

[Recepción: Agosto de 2010 / Conformidad: Setiembre de 2010]

\section{RESUMEN}

El presente artículo tiene por finalidad hacerlos reflexionar sobre la práctica contable en el Perú. No hemos terminado la primera década de este nuevo siglo y nuestra profesión contable, como diría Peter Drucker, se viene caracterizando por los cambios constantes que viene soportando, al grado tal de que ya parece que estamos dentro de nuevos paradigmas. Y lo más importante es que el futuro de nuestra práctica se vislumbra plasmado de cambios constantes, entre otros; debido a la velocidad en la cual viene cambiando la tecnología de la información, la necesidad de la educación continua, de contar con acreditaciones renovables, la manera de interactuar con la alta dirección, de hacer negocios en ambientes globalizados y a gerenciar organizaciones que se reinventan permanentemente. Si a este entorno en la cual se desarrolla la práctica contable en el Perú le agregamos la percepción equivocada que tienen hoy los jóvenes limeños en edad universitaria sobre la profesión contable y los diversos temas que tenemos que solucionar a nivel gremial y con los diversos organismos reguladores de la profesión contable en nuestro país. Y sí a esto le agregamos que la formación de los futuros contadores en las universidades no es la que realmente pide este mercado cambiante.

Todo lo anteriormente descrito nos plantea un gran reto, en la cual todos nosotros, los

\begin{abstract}
The article present to make them reflect on the accounting practice in Peru. We finished the first decade of this new century and our accounting profession, as Peter Drucker would say, has been characterized by constant change has been suffering, to such a degree that now seems that we are in new paradigms. And most importantly, the future of our practice is seen reflected constant changes, among others, due to the speed at which technology is changing the information, the need for continuing education, accreditation of having renewables, way to interact with senior management, doing business in globalized environments and manage organizations that constantly reinvent themselves. Yes to this environment in which accounting practice is developed in Peru we add the misperception that young people today have college-age Lima on the accounting profession and the various issues we have to solve the union level and the various regulatory bodies the accounting profession in our country. And if we add to this the training of future accountants in universities is not the one actually calls this changing market.

Everything described above poses a great challenge, in whichallofus, publicaccountants, regulators of the practice of accounting in our country, including the Peruvian universities have to reinvent the accounting profession to change that perception and make
\end{abstract}

* Docente Auxiliar de la Facultad de Ciencias Contables. Socio de la Firma Auditora URBANO VENTOCILLA \& ASOCIADOS S.C.E-mail: curbano@uhyuvperu.com 
contadores públicos, los organismos reguladores de la practica de la contabilidad en nuestro país, incluyendo las universidades peruanas tienen que reinventar la profesión contable para poder cambiar esa percepción $\mathrm{y}$ hacer que los contadores sigamos siendo considerados por la comunidad de negocios como sus Socios Estratégicos de las Organizaciones, que se caracterizan por crear valor en las organizaciones donde brindan sus servicios profesionales.

Palabras claves: Contabilidad, competitividad, organizaciones y Universidades.

\section{INTRODUCCIÓN}

Hace unos mes terminé de leer un libro que encontré en la Casa de Libro, ubicada en la ciudad de Madrid, titulado La historia de la contabilidad, una traducción al español de la monografía escrita por el Profesor Checo Karl Peter Kheil, titulada "Über einige áltere Berabeitungen des Buchhaltungs-Tractates von Luca Pacioli” escrita en el año 1896 y traducida por el Profesor Mercantil Aragones Fernando López y López en el año 1902. Luego de leerla me quede muy impresionado y gratificado por que había llenado grandes brechas en mi conocimiento acerca de la historia de la partida doble. Sin embargo, la lectura de la citada obra me indujo a reflexionar de cómo nuestra practica profesional había cambiado tanto y que estamos viviendo nuevos paradigmas en la practica de la contabilidad, respecto a lo que deben ser las competencias de un contador para mantenerse competitivo en las organizaciones de este milenio.

Por otro lado, la semana pasada el Rector de la Universidad del Pacífico me invitó a participar en un Foro denominado "El Contador: Socio Estratégico en las Organizaciones" cuyo objetivo era responder a algunas interrogantes en torno al rol del contador en accountants continue to be considered by the business community as its Strategic Partner Organizations, characterized by creating value in organizations where they offer their professional services.

Key words: Accounting, competitiveness, organizations and universities.

la actualidad. En ese Foro escuche que APOYO había efectuado una encuesta en el primer semestre del año 2010 a jóvenes limeños en la cual les preguntaban entre otros cual era su percepción sobre la carrera contable, a lo cual entre otras cosas, ellos respondían ubicándola como una carrera técnica, de nivel intermedio, no gerencial, sustituible con la tecnología de la información y entre las que existen en el medio, ella era la cuarta o quinta opción a escoger (primero estaban; negocios internacionales, economía, administración, etc.). Me quede impresionado con estas respuestas y me pregunté, cuánto de realidad hay detrás de estas respuestas y cuan lejos están de la realidad.

Todo esto me animó a tratar de escribir y compartir con ustedes, como percibo a la práctica contable en nuestro país, de la cual estoy convencido que estamos viviendo una era de cambios, en la cual estoy convencido que las competencias de los contadores tienen que reinventarse constantemente, debido a que los paradigmas ahora son mas frecuentes y lo mas retante es que seguirán cambiando, parafraseando al Profesor Peter Drucker, cuando el decía que esta época en la que nos ha tocado vivir lo único constante es el cambio. 


\section{¿VIVIMOS EN UNA ERA DE CAMBIOS DE NUESTROS PARADIGMAS?}

En la actualidad, se genera un cambio fundamental en la naturaleza y aplicaciones de la tecnología en los negocios, existe un nuevo orden geopolítico, aparecen nuevos ambientes y formas de hacer negocios y empresas. Estos cambios, por citar algunos, tienen implicancias profundas y de gran alcance para nosotros los contadores. Por ejemplo, los cambios y los impactos que se generan en las empresas producto de la evolución desenfrenada de la tecnología de las telecomunicaciones, la integración digital de sonido, datos a imagen, así como la convergencia entre las telecomunicaciones, computación y televisión y si a eso le sumamos las redes digitales de información, conllevara a muchos cambios en la oferta de nuevos y novedosos servicios que a la fecha desconocemos.

Los negocios no se quedan atrás, tienen oportunidades nunca vistas para abrir $y$ aprovechar nuevos mercados, y entre tanto, los mercados tradicionales cambian de manera sustancial, reduciéndose o haciéndose intensamente competitivos. Además, los reducidos márgenes de beneficios, paralelos a las crecientes exigencias del cliente por productos y servicios de calidad, a precios bajos, determinan presiones inexorables en muchas de nuestras empresas. Una realidad apremiante del nuevo ambiente global es la emergencia de una nueva era de competencia más agresiva y sin fronteras en la cual la innovación constante, el aprender a aprender y el convertirse en un proyecto exitoso, son las claves del éxito para mantenernos competitivos.

El futuro trae muchas cosas para todos. Sin embargo, lo más importante que se puede rescatar es la incertidumbre del futuro, lo desconocido, que está en el hoy y mañana. Como lo explica Tom Peters en su libro Re-imagina, "lo desconocido significa la idea esencial de las "formas de vida" en la era de la clonación humana y de la ingeniería genética. Lo desconocido significa la definición de un puesto de trabajo, cuando el software potenciado por la inteligencia artificial tienen más cerebro que tú y yo." Lo desconocido proporciona oportunidades sin igual a quienes sean lo suficientemente atrevidos y decididos para obtener ventaja de ello. Es una era propicia para quienes rompan reglas, para quienes imaginan lo que ha sido imposible hasta ahora ... esto es el comienzo de la nueva empresa, de una nueva forma de pensar y de vivir en estos tiempos de cambios".

En lo que respecta a la profesión contable, podríamos enumerar algunos cambios en nuestros paradigmas, que si los analizamos en su conjunto, podríamos decir que ya estamos viviendo en un entorno de cambios paradigmáticos frecuentes. A continuación citamos algunos de ellos: 


\begin{tabular}{|l|l|}
\hline \multicolumn{1}{|c|}{ ERA } & \multicolumn{1}{c|}{ ES } \\
\hline Prevalece el costo histórico & Prevalecerá el criterio de valor razonable menor \\
Contabilización de futuros & Contabilización de hechos pasados \\
Información a la fecha de corte & Información que ayude a predecir el futuro \\
Principios de contabilidad generalmente aceptados & Marco conceptual, hipótesis y cualidades de la contabilidad \\
Piversos criterios de medición y presentación & Uniformar los criterio de medición y presentación - solo NIIF \\
Mucha incidencia en el registro contable & No existe el concepto extraordinario \\
Poca competencia de las finanzas & Mucha incidencia en el análisis de la información, más allá del simple registro \\
Poca aplicación de la Tecnología de la información & Mucha necesidad de las finanzas \\
Centrado en el propietario & Centrado en el inversionista \\
\hline
\end{tabular}

\begin{tabular}{|l|l|}
\hline \multicolumn{1}{|c|}{ ERA } & \multicolumn{1}{|c|}{ ES } \\
\hline Muy prudente & Menos prudente \\
Prevalece el criterio tributario & Prevalece el criterio financiero \\
Importa lo tangible & Lo intangible tiene mayor valor \\
Provee información histórica & Provee información estratégica \\
Revelaciones insuficientes & Más revelaciones y análisis de riesgos y de sensibilidad \\
No comparabilidad & Sí a la comparabilidad \\
Brindar información contable & Crear valoren las organizaciones \\
\hline
\end{tabular}

\section{¿CÓMO AFECTA LO ANTERIOR A NUESTRA PRÁCTICA EN NUESTRO MEDIO?}

Por un lado, el entorno, la tecnología de la información, la forma de hacer empresa hoy, y las nuevas organizaciones en un ambiente globalizado, mercados abiertos y altamente competitivos, nos complican más el reto de ser competitivos. Para serlo debemos estar aprendiendo e innovando constantemente nuestros conocimientos, habilidades y destrezas gerenciales a la velocidad en la cual cambia el entorno en la cual nos desenvol- vemos. Por otro lado, no podemos negar la brecha que existe entre las competencias que hoy necesitan y aprenden nuestros futuros contadores en las universidades peruanas ylo que realmente requiere las empresas que trabajan en nuestro país. Esto también impacta de igual manera a los catedráticos universitarios, si no son o se capacitan constantemente en aspectos de las competencias necesarias para la profesión contable así como en el manejo de nuevas herramientas y nociones de pedagogía. Al respecto, también debemos re-enfocar la manera de enseñar y/o de pre- 
parar a los alumnos. Debemos preparar a los alumnos para un futuro desconocido, ambiguo y rápidamente cambiante, lo que significa que "aprender a aprender" es mucho más importante que dominar una materia para pasar los examenes.

Este entorno ha eliminado una gran parte de la práctica contable legada por Fray Luca de Paciolo, que era la de los registradores, la tecnología de información se encargó de esto. Ahora, de su legado nos queda la parte normativa, en otras palabras los aspectos técnicos de nuestra profesión, la cual se puede resumir en las Normas Internacionales de Información Financiera - NIIF, que hoy se ha convertido en el lenguaje de las grandes Corporaciones que cotizan en las diversas Bolsas de Valores del mundo. Sin olvidarnos de las NIIF para PYMES que será la relevante para las demás empresas. Un primer reto es que todos lo contadores conozcan, y sepan aplicar correctamente estas normas técnicas en sus empresas, de tal manera de asegurar que la información que están presentando a la Alta Dirección sea pertinente para la toma de decisiones. Esto debe ser el punto de partida para la reinvención de nuestra práctica contable actual.

$\mathrm{Si}$ a lo dicho anteriormente le sumamos el hecho de que en nuestro país según una encuesta efectuada por APOYO en el primer semestre del 2010 a jóvenes en edad universitaria, a los cuales se les preguntó cual era su percepción sobre la carrera de contabilidad, respondiendo que era una carrera técnica, fácilmente reemplazable por los software contable, una carrera que difícilmente en una organización llegaría a niveles gerenciales, prefiriendo a la carrera de contabilidad, la de negocios internacionales, economía, administración, etc. El efecto de esta percepción, en las universidades particulares donde estudian los jóvenes que provienen de los estratos sociales A y B, ha sido en promedio una reducción del $40 \%$ en el número de postulantes en relación con años anteriores. Esta percepción felizmente no se condice con la realidad en su totalidad en nuestro país, sin embargo es una muestra más del problema a futuro que podría tener la práctica de nuestra profesión en nuestro país. De no revertir esta percepción equivocada, ésta podría estar afectando a nuestros futuros talentos que son los jóvenes en edad universitaria, de poner escoger estudiar nuestra profesión y ser los sucesores nuestros en el futuro mediato.

Por otro lado, en nuestro país nuestra práctica contable necesita mejorar y gran parte de ello esta en nuestras manos, no podemos depender de otros, se trata de nuestro futuro, de nuestras vidas y de nuestras familias. Es importante que nos demos cuenta de que si nosotros no somos los promotores del cambio del statu quo que atraviesa nuestra profesión contable en el Perú, podemos poner en serio riesgo nuestra práctica profesional. No es suficiente ser espectadores pasivos de lo que está pasando con nuestra práctica contable. Quizás, el mayor problema que tenemos es encontrar un tiempo para poder hacerlo y perder el miedo a las críticas, $y$ si es necesario recurrir a las instituciones de la sociedad civil para que nos ayuden en esta tarea, evaluémosla, que quizás pueda ser una alternativa interesante al respecto. Es necesario que nuestros contadores de hoy y los próximos que se formen tomen conciencia de esta gran amenaza que trae el entorno de hoy para nuestra práctica profesional, estando obligados a convertirla en una gran oportunidad para re-inventar nuestra profesión en todo aspecto, sea de forma $\mathrm{y} / \mathrm{o}$ fondo.

A continuación describimos a manera de ejemplo tres situaciones que afectan de manera negativa la práctica contable de nuestro país: i) Los reguladores de la profesión con- 
table en nuestro país, no norman en forma coordinada, ni a la velocidad que lo pide la organizaciones de hoy en día. No hay un norte estratégico definido de la profesión contable a nivel país. Por citar un ejemplo, después de casi tres años de espera, recién hace un mes se hayan aprobado una serie de normas contables internacionales modificadas y emitidas a nivel internacional. ii) el regulador que tiene más ingerencia en nuestros colegas de la profesión es el encargado de la administración tributaria de nuestro país. iii) La existencia de dos Colegios de Contadores en Lima, que operan a la vez, hace más de tres años, que están más preocupados por atender las demandas judiciales que se hacen entre ellos, afectando el cumplimiento de su misión gremial, que entre otros es capacitar a los contadores y ser un ente representativo de la sociedad civil en nuestro medio.

Lo mencionado anteriormente viene contribuyendo a que gran parte del gremio contable, sobre todo aquellos cuyas edades bordean los 40 años o más, poco a poco se vean amenazado de no ser competitivos por falta de educación continuada. Esto último podría incluso afectar este gran momento que vive nuestro país a nivel mundial, la imagen de ser un país emergente, atractivo a la inversión extranjera, las grandes proyecciones de inversión y de ingresos de divisas en los sectores de la minería, gas, pesca, inmobiliario, agroindustrial, entre otros. Que pasará cuando vengan estos inversionistas y constituyan sus empresas en nuestro país y requieran contratar contadores profesionales competitivos a nivel internacional, y no los encuentren en el mercado local, tendrán que recurrir a contadores de otros países o de sus Casas Matrices. Qué pasará con nuestros jóvenes profesionales contables que egresan de nuestras universidades, que salen con una formación profesional que no condice con las competencias que necesitan las organizaciones de hoy, para incorporarlos. Las mallas curriculares no contemplan la inclusión de cursos relacionados con competencias muy necesarias que deben ser evaluadas constantemente. Cursos relacionados con las finanzas corporativas, tecnología de la información en los negocios, ética profesional, comportamiento organizacional, idiomas, estrategias organizacionales, destrezas gerenciales, análisis cuantitativos de negocios, matemática financiera y estadística, entre otros.

Tenemos que darnos cuenta que hoy día la formación del contador necesita de una reforma integral orientada a formar un contador competitivo que forme parte del equipo gerencial en las organizaciones de hoy.

\section{CONCLUSIONES}

Las implicancias del cambio de paradigmas en la contabilidad, implicará sacrificios de contenidos intelectuales y continuidades paradigmáticas dentro de la nueva ciencia contable, la determinación de contenidos inconmensurables partirá del acuerdo o concertación de la comunidad científica contable en armonía con lo que requiere el mercado laboral.

La economía actual se caracteriza por haber propiciado la innovación y desarrollo de la tecnología de la información, lo que ha revolucionado a las organizaciones y a los individuos en sus formas de pensar, ser y hacer. Los contadores debemos convertirnos es parte del equipo gerencial de las organizaciones donde laboramos y con la misión de crear valor.

La tecnología de la información, en sus múltiples expresiones, propició la globalización económica de los negocios en donde se modifican los esquemas tradicionales de abastecimiento, producción y distribución, 
provocando niveles agudos de competencia entre las organizaciones. El proceso de toma de decisiones aparecerá vacío sin el aporte de la contabilidad como un sistema de información analizado y proyectado.

La contabilidad debe ser parte proactiva e integral, puesto que su producto, conceptualmente hablando, es la información financiera como base para ejercer control, dirección y fomento de la toma de decisiones como un proceso descentralizado.

Vivimos en una era de cambios, que afecta el modo de hacer empresa y gerenciarla, y que también cambian nuestros paradigmas contables, razón por la cual todos los profesionales de la contabilidad debemos tener la competencia de la educación continua y si nos es posible, estar inscritos en los Organismos Internacionales que norman la profesión contable a fin de no desactualizarnos.

Las universidades deberían definir el perfil del contador que quieren formar, el cual debe ser competitivo para las organizaciones que lo requieran. Para esto deben constante- mente evaluar y cambiar su malla curricular, incorporando todas las competencias que sean necesarias para que los futuros contadores sean competitivos.

Es imperativo solucionar el problema actual del gremio contable y cambiar la percepción que tiene los jóvenes sobre la profesión contable. Al respecto, debería formarse un grupo de representantes del gremio contable para que coordine con los organismos reguladores de la profesión a fin de poder logar que estos trabajen a la velocidad necesaria para lograr mantener la competitividad de la profesión contable en el Perú.

\section{REFERENCIAS BIBLIOGRÁFICAS}

1. LÓPEZ Y LÓPEZ, Fernando (2004). Historia de la contabilidad. Madrid.

2. TAPSCOTT, Don y CASTON, Art (1995). Cambios de paradigmas empresariales. Colombia.

3. PETERS, Tom (2003). Re-imagina. Madrid. 